\title{
Caracterización clínica y etiología de baja visión y ceguera en una población adulta con discapacidad visual
}

\section{Clinical characteristics and etiology of low vision and blindness in an adult population with visual impairment}

\author{
Johana Arias-Uribe ${ }^{1,2}$, Yuliana Llano-Naranjo ${ }^{1,2}$, Esau Astudillo-Valverde ${ }^{1,3}$ y Juan C. Suárez-Escudero ${ }^{1,4 *}$

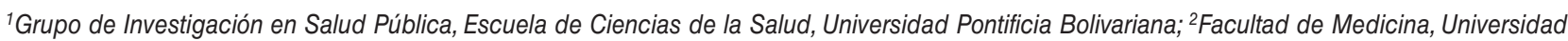 \\ Pontificia Bolivariana; ${ }^{3}$ Clínica de Oftalmología Sandiego; ${ }^{4}$ Facultad de Medicina, Universidad Pontificia Bolivariana. Universidad Centros de Estudios \\ en Salud (CES), Institución Universitaria Instituto Tecnológico Metropolitano (IMT) e Institución Prestadora de Salud (IPS) Solaz, Medellín, Colombia
}

\section{Resumen}

Introducción: Baja visión y ceguera conforman la discapacidad visual, uno de los tipos de discapacidad con mayor prevalencia mundial y, al parecer, el de mayor frecuencia en Colombia. La etiología convencional de las deficiencias permanentes y transitorias responsables de discapacidad visual como el glaucoma, la catarata, la ceguera infecciosa y las ametropías no corregidas puede estar cambiando por la presencia de otras entidades y los resultados de los programas de salud pública, y es necesario extender el espectro etiológico tanto a causas oftalmológicas como a no oftalmológicas. Objetivos: Identificar la etiología y las características clínicas más relevantes en una población adulta con discapacidad visual. Materiales y métodos: Estudio transversal descriptivo realizado en 96 adultos con discapacidad visual confirmada. Resultados: El $61 \%$ de los pacientes presentaban baja visión. En el $55 \%$ de los casos la etiología de la deficiencia visual se ubicaba en el segmento posterior; en el 21\%, en el segmento anterior, y en el 10\%, en la vía y/o corteza visual. Las enfermedades individuales más frecuentes fueron: miopía patológica (MP), neuropatía óptica (NO) no glaucomatosa, glaucoma y degeneración macular relacionada con la edad (DMRE). Conclusiones: En este estudio las enfermedades con compromiso del segmento posterior ocuparon las etiologías más frecuentes relacionadas con discapacidad visual, y las entidades neurooftalmológicas y el trauma ocular se identificaron como una potencial fuente de deficiencias visuales. Es necesario continuar realizando estudios que esclarezcan si el espectro de etiologías convencionales causantes de discapacidad visual ha cambiado, con el objetivo de redireccionar los programas de promoción, prevención, diagnóstico, tratamiento y rehabilitación en baja visión y ceguera.

Palabras clave: Etiología. Baja visión. Ceguera. Personas con daño visual. Estadísticas de secuelas y discapacidad.

\section{Abstract}

Introduction: Low vision and blindness make up the visual disability, one of the types of disability with the highest worldwide prevalence, and apparently the most frequent at a Colombian level. The conventional etiology of permanent and transitory deficiencies responsible for visual impairment such as glaucoma, cataract, infectious blindness and uncorrected ametropias, may be changing due to the presence of other entities and results of public health programs, and it is necessary to extend the etiological spectrum to both Ophthalmological causes as not ophthalmological. Objectives: To identify the

Correspondencia:

*Juan Camilo Suárez-Escudero

Lab. de Anatomía Humana Bernardo Gallego

Campus Robledo C. 78 B No. 72A $109 \quad$ Fecha de recepción: 08-08-2017

Medellín, Colombia $\quad$ Fecha de aceptación: 27-04-2018

DOI: 10.24875/RMO.M18000033

Disponible en internet: 02-07-2018 Rev Mex Oftalmol. 2018;92(4):201-208 www.rmo.com.mx the CC BY-NC-ND license (http://creativecommons.org/licenses/by-nc-nd/4.0/). 
etiology and the most relevant clinical characteristics in an adult population with visual impairment. Materials and methods: Descriptive cross-sectional study in 96 adults with confirmed visual impairment. Results: $61 \%$ with low vision; in $55 \%$ the etiology of the visual deficiency was in the posterior segment, $21 \%$ in the anterior segment and $10 \%$ in the pathway and/or visual cortex. The most frequent individual pathologies: pathological myopia, non-glaucomatous optic neuropathy, glaucoma and macular degeneration related to age. Conclusions: in this study, pathologies with posterior segment involvement occupied the most frequent etiologies related to visual disability, and the neuro ophthalmological and ocular trauma entities were identified as a potential source of visual deficiencies. It is necessary to continue carrying out studies that clarify whether the spectrum of conventional etiologies causing visual disability has changed, with the aim of redirecting programs of promotion, prevention, diagnosis, treatment and rehabilitation in low vision and blindness.

Key words: Etiology. Low vision. Blindness. Visually impaired persons. Statistics on sequelae and disability.

\section{Introducción}

Los conceptos y definiciones sobre la discapacidad cambian con el tiempo ${ }^{1}$ y se adaptan cada vez más a un contexto intersectorial, más allá del mero sector salud. Desde la óptica del funcionamiento humano por parte de la Organización Mundial de la Salud (OMS), la discapacidad es universal, forma parte de la condición humana y no es un fenómeno netamente biológico ni tampoco exclusivamente social ${ }^{2}$. La discapacidad es una condición que pone en diálogo y continua interacción lo corporal (déficits), lo individual (limitaciones en la actividad y factores personales) y lo social (restricciones en la participación y factores ambientales) ${ }^{3,4}$. En este contexto se recomienda comprender la discapacidad visual, que a partir del déficit visual se clasifica en baja visión o ceguera, y las personas pueden tener un amplio rango de limitaciones en la actividad, restricciones en la participación, barreras, facilitadores personales y ambientales.

Desde el año 2011 la OMS reporta una prevalencia mundial de discapacidad del 15\% (cerca de un billón de personas con algún tipo de discapacidad) ${ }^{3}$. En el año 2011 una revisión sistemática con datos de 20 años (1990-2010) reportó una prevalencia cercana a los 223 millones de personas con discapacidad visual, distribuidas en 32.4 millones de personas invidentes y 191 millones de personas con baja visión $n^{5}$. Las cifras actuales de la OMS indican una prevalencia mundial de discapacidad visual de 253 millones $^{6}$, de los cuales 217 millones tienen baja visión y 36 millones son invidentes. La baja visión es más frecuente que la ceguera. La discapacidad visual como una forma de discapacidad por deficiencia sensorial constituye cerca del $28 \%$ de todas las discapacidades, y es así una de las deficiencias con limitaciones en la actividad y restricciones en la participación (discapacidad) más prevalentes.

En Colombia, según datos oficiales a partir del censo nacional del año 2005, la prevalencia general de discapacidad visual (desde una óptica de limitaciones permanentes para ver, a pesar de usar lentes 0 gafas) es del $2.72 \%$ (cerca de 1,121,129 personas) ${ }^{7,8}$, dato que constituye el $43.4 \%$ dentro de la población colombiana con alguna discapacidad ${ }^{8}$. En otros términos, la deficiencia más frecuente en la población con discapacidad en Colombia es la visual (hasta que se publiquen los datos del censo poblacional colombiano del año 2018).

Las etiologías más frecuentes de las deficiencias visuales que conducen a una condición de baja visión o ceguera son, en general, de origen oftalmológico. La OMS señala que en el mundo los errores de refracción no corregidos (miopía, hipermetropía y astigmatismo) producen discapacidad visual en un $43 \%$ de casos; las cataratas no operadas, en un $33 \%$, y el glaucoma, en un $2 \%{ }^{6}$. En Latinoamérica, a partir del informe oficial del programa Visión 2020 del año 2013, las causas principales de baja visión en personas mayores de 50 años fueron las cataratas (40-76\%), los errores refractivos (18-50\%) y causas ligadas al segmento posterior $(2-23 \%)^{9}$. Se trata de datos con gran variabilidad porque incluyen múltiples estudios, algunos realizados sólo con población urbana o rural y otros con ambos tipos (Argentina, Chile, Perú, Guatemala, Paraguay). Dicho informe no incluyó a Colombia por ausencia de información ${ }^{9}$.

Sin embargo, médicamente existen múltiples etiologías oftalmológicas y no oftalmológicas (neurológicas y sistémicas, por ejemplo) que pueden instaurar deficiencias visuales permanentes, causantes también de discapacidad visual. Es posible que la etiología convencional (netamente oftalmológica) de la discapacidad visual esté cambiando por fenómenos de transición epidemiológica, el incremento en la prevalencia de las enfermedades crónicas no transmisibles, la instauración y finalización de planes de promoción y prevención instaurados por la OMS y la Organización Panamericana de la Salud (OPS) (programas de control de 
ceguera infecciosa, por ejemplo), más la dinámica actual de los planes de atención básica en salud, y la cobertura en los sistemas de salud. Son útiles los estudios que exploran las características clínicas y etiológicas a nivel local, regional y nacional. En el caso colombiano, son escasos los estudios sobre la etiología y las características clínicas de las personas con discapacidad visual, pero se requieren para redireccionar los programas de promoción, prevención y asistencia en baja visión y ceguera.

\section{Objetivo}

Identificar y clasificar la etiología y las características clínicas más relevantes en una población adulta con discapacidad visual de la ciudad de Medellín (Colombia). Los datos locales y nacionales al respecto son escasos y se basan en proyecciones de estudios realizados en otros países y contextos. Otras etiologías, diferentes a las oftalmológicas convencionales, pueden estar dinamizando el panorama de discapacidad visual.

\section{Métodos}

Estudio transversal descriptivo realizado en 96 personas mayores de 18 años con discapacidad visual identificadas por el Grupo de Discapacidad Visual de la Escuela de Ciencias de la Salud (ECS) de la Universidad Pontificia Bolivariana (UPB) de la ciudad de Medellín (Antioquia, Colombia). Muestra a conveniencia por dificultades en la identificación de personas con discapacidad visual confirmada dentro del sistema de salud y en la práctica profesional, secundarias a barreras como poca sensibilización y capacitación técnica en el tema, dificultades en la codificación diagnóstica con la CIE-10R y poca visibilidad de las personas con discapacidad visual dentro del sistema de salud. Las personas fueron revisadas en 16 jornadas de investigación en las instalaciones de la ECS-UPB, en el marco de un estudio clínico de la línea de investigación en discapacidad, por parte del Grupo de Investigación en Salud Pública ECS-UPB, realizado en el segundo semestre del año 2015. Se recolectaron datos sociodemográficos, información clínica sobre etiología y comorbilidades, toma de agudeza visual (AV) con cartilla ETDRS y aplicación de un formato adaptado de la Clasificación Internacional de Funcionamiento, Discapacidad y Salud (CIF-2001).

Se categorizó el déficit visual en los pacientes con discapacidad visual mediante la clasificación de severidad del déficit visual WHO consultation CIE-10 ${ }^{10}$. LOS criterios empleados de deficiencia visual moderada-severa (para clasificar en baja visión o ceguera) por AV mediante la cartilla ETDRS fueron los siguientes:

- Baja visión: AV corregida inferior o igual a 6/18 (0.3 en notación decimal $0 \leq 20 / 60$ en notación americana) y/o campo visual menor a $20^{\circ}$ desde el punto de fijación en el mejor ojo, después del mejor tratamiento, refracción común o ambas cosas, pero con la existencia aún de un resto visual potencialmente utilizable para la planificación y ejecución de tareas (en esta definición está incluido el término de $\mathrm{AV}$ funcional).

- Ceguera: AV menor a 3/60 (0.05 en notación decimal $0<20 / 400$ en notación americana) en el mejor ojo con la mejor corrección posible y/o campo visual menor a $10^{\circ}$ desde el punto central de fijación ${ }^{5,10,11}$.

Las características clínicas consideradas en el estudio fueron: edad, sexo, nivel educativo y alfabetismo, categoría de discapacidad visual, diagnóstico etiológico oftalmológico y/o neurooftalmológico, edad de inicio de los síntomas visuales y utilización de dispositivos no ópticos y ópticos para compensar el déficit visual. La etiología del déficit visual responsable de la discapacidad visual se agrupó por ubicación anatómica en segmento anterior, segmento posterior, vía y corteza visual, y ubicación mixta.

En este estudio no se utilizó la encuesta Rapid Assessment of Avoidable Blindness (RAAB) porque está restringida a los mayores de 50 años, y su objetivo es estimar la prevalencia de ceguera y baja visión en una población evaluando la cobertura y calidad de las cirugías de catarata.

Las variables cualitativas se reportaron con frecuencia y porcentaje; en las variables cuantitativas se evaluó si seguían una distribución normal, mediante la prueba de Kolmokorov-Smirnov (prueba KS). Según esto, se obtuvieron medias o medianas con su respectiva desviación estándar (DE) o rango intercuartil.

Estudio aprobado por comité de ética e investigación de la ECS-UPB. Los procedimientos utilizados en los pacientes se realizaron tras una explicación y obtención del consentimiento informado. Fue un proyecto financiado por el Centro Integrado para el Desarrollo de la Investigación (CIDI) de la UPB, con sede en Medellín.

\section{Resultados}

Hubo 96 pacientes con discapacidad visual confirmada mediante criterios de AV en el mejor ojo con la 
mejor corrección y/o campimetría. La edad promedio fue de 50.5 años (DE: 19.5), siguiendo una distribución normal (prueba KS: $p=0.806)$. El $57 \%$ (55/96) fueron mujeres. El 33\% (32/96) poseían nivel educativo de primaria (primaria incompleta: $16.7 \%$; primaria completa: $14.6 \%$, y primaria en curso: $2.1 \%)$; el $30 \%$ (29/96), nivel educativo de secundaria (secundaria incompleta: $13.5 \%$; secundaria completa: $12.5 \%$, y secundaria en curso: $4.2 \%$ ). La prevalencia de analfabetismo fue del $15 \%(14 / 96)$.

Hubo un 61\% (59/96) de pacientes con baja visión y un $39 \%$ (37/96) de pacientes con ceguera. La tabla 1 detalla la frecuencia de los estadios visuales del déficit en los pacientes, según la clasificación de severidad del déficit visual WHO consultation CIE-10 ${ }^{10}$.

Los diagnósticos individuales, a nivel oftalmológico y neurooftalmológico, más frecuentes en los pacientes fueron: MP (16\% 15/96]), NO (10\% 10/96]), glaucoma (9\% 9/96]), DMRE (9\% 9/96]), retinosis pigmentaria (RP) $(7 \% 7 / 96])$, trauma ocular (7\% $7 / 96])$, catarata (6\% 6/96]), ametropías no corregidas (4\% 4/96]), albinismo ocular $(2 \% 2 / 96])$ y coroideremia $(2 \% 2 / 96])$. La figura 1 muestra la distribución de los diagnósticos agrupados por ubicación anatómica.

El segmento posterior del globo ocular, conformado por la retina, la coroides y la cámara vítrea, fue el sitio anatómico más afectado por los diagnósticos individuales identificados en todos los pacientes.

El inicio de los síntomas visuales, en el 76\% (73/96) de los casos, fue posterior al nacimiento, a los 28.8 años (DE: 22.7) de promedio, y sigue una distribución normal según la prueba KS ( $p=0.095)$. En el $24 \%$ (23/96) de los pacientes el inicio de los síntomas se presentó en el nacimiento, es decir, que la dificultad visual era congénita.

El $67 \%(64 / 96)$ y el $43 \%(41 / 96)$ de los pacientes utilizaban dispositivos no ópticos y ópticos, respectivamente, para compensar su déficit visual. La tabla 2 muestra en detalle la frecuencia de uso de dispositivos ópticos (telescopios y lupas) y no ópticos (bastón, Braille y perro guía) en los pacientes.

\section{Discusión}

La discapacidad visual produce cambios y adaptaciones en varios aspectos del funcionamiento ${ }^{12}$ de las personas, al ser una temática con diferentes retos intersectoriales que trascienden la atención en salud ${ }^{13-15}$.

En este estudio se encontraron más pacientes con baja visión que con ceguera (61 vs. 39\%; razón: por cada invidente hay cerca de dos pacientes con baja
Tabla 1. Frecuencia de los estadios visuales del déficit visual en los pacientes

\begin{tabular}{|c|c|c|}
\hline Estadio visual & n & 0 \\
\hline Deficiencia visual moderada $(<20 / 60-20 / 200)^{*}$ & 42 & N \\
\hline Deficiencia visual severa $(<20 / 200-20 / 400)^{*}$ & 17 & 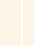 \\
\hline Ceguera $(<20 / 400)^{*}$ & 37 & $£$ \\
\hline *Mediante toma de AV. & & (c) \\
\hline
\end{tabular}

Tabla 2. Frecuencia de uso de dispositivos ópticos y no ópticos

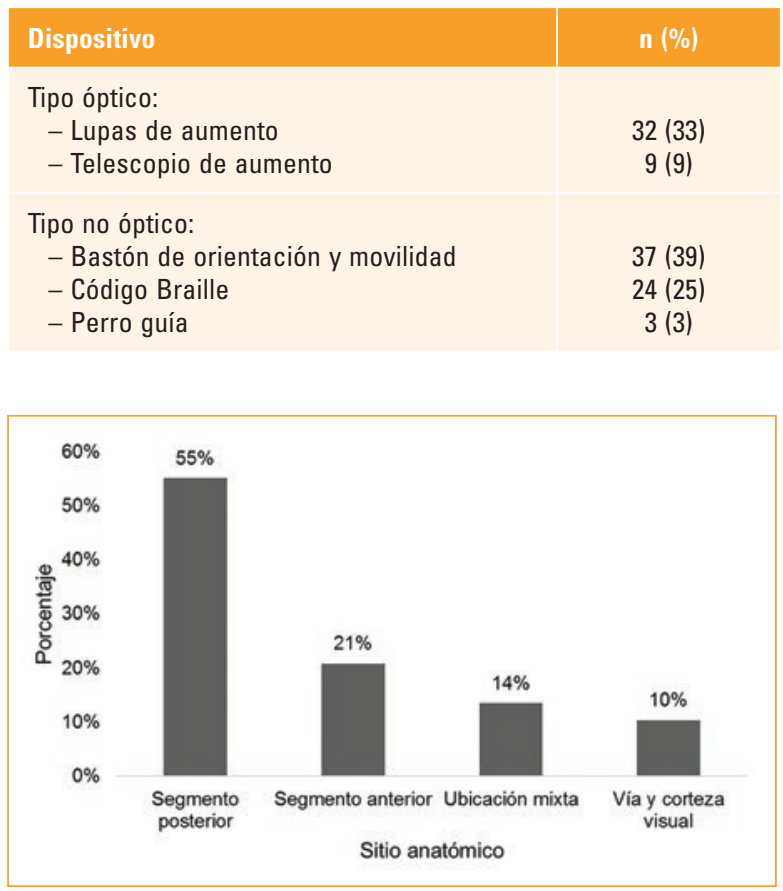

Figura 1. Diagnósticos agrupados por ubicación anatómica.

visión), y entre los de baja visión la categoría de déficit más frecuente fue la deficiencia visual moderada (AV de entre 20/60 y 20/200). En América Latina y el Caribe se calcula que por cada millón de habitantes cerca de 20,000 tienen baja visión y 5,000 son invidentes ${ }^{16}$, y a nivel mundial se estima una razón entre ceguerà y baja visión de 1:6 $6^{6}$.

Los resultados sobre la etiología de la deficiencia visual causante de discapacidad visual, en el presente estudio, muestran un gran espectro de enfermedades capaces de afectar al funcionamiento visual de manera permanente. Las enfermedades individuales con una frecuencia mayor al 5\% fueron la MP, la NO no glaucomatosa, el glaucoma, la DMRE, la RP, el trauma 
ocular y la catarata. No se encontraron casos de discapacidad visual (en especial, ceguera) de origen infeccioso, y las causas de mayor prevalencia de discapacidad visual reportadas a nivel mundial, como la catarata y el glaucoma, no ocuparon los primeros puestos en esta población estudiada ${ }^{6}$.

Por ubicación anatómica, las enfermedades del segmento posterior fueron las más relevantes con una frecuencia del 55\%. En países de ingresos altos la principal causa de discapacidad visual son los defectos refractivos no corregidos, la DMRE, el glaucoma y la retinopatía diabética ${ }^{17}$, y en países de ingresos bajos (donde se ubica Colombia) la catarata no operada domina como la principal causa de ceguera (51\%), seguida del glaucoma $(8 \%)$ y la DMRE $(5 \%)^{18}$. El plan Visión 2020 del año 2013 reportó como causas principales de baja visión en personas mayores de 50 años las cataratas (40-76\%), los errores refractivos (18-50\%) y causas ligadas al segmento posterior $(2-23 \%)^{9}$. Los autores de este trabajo no encontramos otras publicaciones sobre el impacto de la MP y la NO en la baja visión o la ceguera en Colombia.

En el año 2015 nuestro grupo reportó un hallazgo similar (baja frecuencia relativa del glaucoma y la catarata) en cuanto a causas de discapacidad visual identificadas mediante la revisión y análisis de 107 historias clínicas de pacientes atendidos en dos centros de referencia de la ciudad de Medellín entre los años 2011 a 2013; la catarata representó el 4.5\% y el glaucoma crónico avanzado sólo el $2.2 \%$ de las causas de discapacidad visual ${ }^{19}$.

Si bien en este estudio no se encontró la catarata como la principal causa de discapacidad visual, es claro que en Latinoamérica ocupa alrededor del 38$74 \%$ de las causas evitables de ceguera ${ }^{20}$.

En el presente estudio llama la atención la presencia de la MP como etiología de discapacidad visual. La MP se ha definido de diversas maneras en la literatura oftalmológica. Algunos la consideran como un error refractivo mayor de -6.00 dioptrías $^{21}$. Yasuo Tano la define como toda miopía asociada a cambios degenerativos del segmento posterior ${ }^{22}$ y la Academia Americana de Oftalmología la define como un error refractivo mayor de -6.00 dioptrías con una longitud axial mayor de $26.5 \mathrm{~mm}^{23}$. Un metaanálisis del año 2015 la definió como ojos con atrofia coriorretiniana igual o más severa que una atrofia difusa ${ }^{24}$. En términos clínicos, la MP constituye una entidad refractiva y degenerativa. Se reporta como una de las principales causas de discapacidad visual en el este de Asia $(12-27 \%)^{24,25}$ y una de las primeras causas de discapacidad visual en la población europea (se reporta una prevalencia del $7 \%)^{26}$. En Colombia no existen reportes ni datos respecto a la epidemiología e implicaciones de la MP como causa de discapacidad visual.

La DMRE como entidad degenerativa crónica de la mácula es una causa de discapacidad visual que ha venido en aumento en las últimas décadas y afecta principalmente al campo de visión central. Es la principal causa de ceguera en la población adulta de los países occidentales industrializados ${ }^{27,28}$, y la tercera causa de discapacidad visual actual a nivel mundial ${ }^{28}$. La prevalencia mundial de DMRE es cercana a 170 millones de personas ${ }^{28}$. La OMS estima que la población con DMRE se duplicará en el año 2020 (principalmente en los países industrializados) ${ }^{10}$. Wong, et al. proyectan para el año 2040 una prevalencia mundial de DMRE de 288 millones de personas ${ }^{29}$. Si la catarata no operada y la ceguera infecciosa (tracoma y oncocercosis) continúan siendo los principales retos en salud visual en varios países no industrializados, la DMRE constituye hoy día el mayor reto en salud visual y bienestar en los países industrializados. En Latinoamérica hay diferentes reportes sobre la magnitud e importancia de la DMRE. En un estudio brasileño con tres millones de personas con déficit visual la DMRE fue responsable del $5.46 \%$ de los $\operatorname{casos}^{30}$. En Ecuador, en un estudio con 802 pacientes, la DMRE causó ceguera en el $3 \%$ de los $\operatorname{casos}^{31}$. En Colombia no hay datos oficiales sobre la magnitud epidemiológica y clínica de la DMRE y la discapacidad visual; sin embargo, en el presente estudio la DMRE fue la cuarta enfermedad más frecuente en los pacientes.

En Inglaterra se reportan las enfermedades hereditarias de la retina como las principales causas de ceguera en la población con edad comprendida entre los 16 y los $64 \operatorname{años}^{32}$. La prevalencia mundial de RP reportada es de $1: 4,000^{33}$, cerca de 1.5 millones de personas. En este contexto, la RP es la degeneración hereditaria más frecuente de la retina, primera causa de ceguera genética en adultos, caracterizada por nictalopía, pérdida progresiva de los campos visuales periféricos, compromiso de fotorreceptores y atrofia retiniana ${ }^{32,33}$. La RP se transmite con patrón autosómico recesivo con una frecuencia de entre el 50 y el $60 \%$ de los casos, con patrón autosómico dominante en el $30-40 \%$ y ligado al $X$ en el $5-15 \% 33,34$. La RP fue la quinta enfermedad individual en los pacientes con discapacidad visual aquí estudiados. En Colombia no hay estudios con suficiente poder estadístico que permitan comprender la importancia de la RP y otras causas hereditarias de la retina en la discapacidad visual. 
En Colombia se han realizado algunos estudios sobre causas de discapacidad visual. En el departamento del Caquetá (año 2004), en una muestra aleatoria de 10,111 personas, la catarata contribuyó a más de la mitad de las causas de ceguera $(54.7 \%)$, los errores refractivos no corregidos contribuyeron en un $11.9 \%$ y el glaucoma ocupó el $4.76 \%$ de las causas ${ }^{35}$. En el departamento de Santander, en un estudio publicado en el año 2009, el glaucoma ocupó el $2.8 \%^{36}$. En 213 personas con limitación visual en la ciudad de Bogotá se reportó una prevalencia de retinopatía diabética del $18.3 \%{ }^{37}$. En la ciudad de Cali una caracterización de 124 niños en condición de discapacidad visual reportó como causas más frecuentes de ceguera infantil (potencialmente prevenible) la retinopatía del prematuro (33.8\%), la anomalía de la vía visual $(20.9 \%)$ y la toxoplasmosis ocular $(15.3 \%)^{38}$. Sin embargo, la ausencia de una adecuada sistematización etiológica y clínica de la población con discapacidad visual en Colombia (con información más clínica y específica de la que arroja la localización y caracterización de población con discapacidad del Ministerio de Salud) dificulta una verdadera comprensión académica y práctica sobre el tema. Esta conclusión es similar a la realizada por Cañón Cárdenas en el año $2011^{39}$, cuando revisó los datos poblacionales de baja visión en Colombia.

Llama la atención que en nuestro estudio otras enfermedades y entidades fuera del segmento posterior y anterior del globo ocular, como el trauma ocular y enfermedades de la vía y corteza visual, pueden producir deficiencias visuales permanentes en rango baja visión o ceguera. La OMS reporta al menos 1,000 millones de personas con algún trastorno neurológico ${ }^{40}$, y la magnitud o repercusión de las deficiencias visuales de origen neurológico como la NO no glaucomatosa, así como el compromiso vascular, neurodegenerativo o desmielinizante de áreas cerebrales visuales, son retos actuales por estudiar en la temática de la discapacidad visual.

La edad es una variable cada vez más documentada para comprender la etiología y epidemiología de la discapacidad sensorial (visual y auditiva) y mental ${ }^{3}$. El informe mundial de discapacidad del año $2011^{3}$ indica que una de cada siete personas con edad mayor de 15 años posee algún tipo de discapacidad. Dicha relación con la edad se ha evidenciado también en la población con discapacidad visual: una de cada tres personas mayores de 65 años puede tener baja visión, la cual es una causa común del deterioro crónico del funcionamiento en las personas mayores de 70 años ${ }^{41}$, y cerca del $65 \%$ de toda la población mundial con discapacidad visual tiene una edad mayor o igual a 50 años $^{42}$. En este estudio la edad promedio de los pacientes con discapacidad visual fue de 50.5 años.

La OMS reporta desde el año $2011^{3}$ una mayor tendencia en las cifras de discapacidad hacia el género femenino. En la población de estudio, el género femenino presentó una frecuencia ligeramente mayor (57\%). Varios estudios descriptivos en discapacidad visual han reportado una mayor prevalencia en mujeres ${ }^{17,43}$, y dicha disparidad se acentúa en países de altos ingresos cuando se trata de discapacidad visual tipo ceguera $^{42}$. Stevens, et al. reportan que el $60 \%$ de las personas invidentes y el $57 \%$ de las personas con baja visión son mujeres ${ }^{14}$; aunque los datos aún son limitados para explicar dicha tendencia, se relaciona en parte con la mayor esperanza de vida de las mujeres ${ }^{44}$. En Colombia, las cifras del censo poblacional de 2005 describen que la deficiencia visual afecta más al género masculino en edades que van desde el nacimiento hasta los 14 años, mientras que las mujeres se ven más afectadas en la adultez y la vejez, y esta diferencia se ve más acentuada después de los 34 años?.

En nuestro estudio se encontró una frecuencia de analfabetismo del $15 \%$, y algunos pacientes estaban cursando estudios de primaria y secundaria ya siendo adultos. El Latino Eye Study ${ }^{45}$, un estudio transversal descriptivo realizado en 2004 en una población de 6,357 pacientes, reportó que tener 12 o más años de educación, comparado con 0-6 años, impacta positivamente en la calidad visual de los pacientes. Otros autores han observado que la prevalencia de discapacidad visual aumenta en algunos sectores con poblaciones caracterizadas por bajos niveles culturales y educativos ${ }^{46}$.

Un facilitador en discapacidad visual consiste en la asistencia a servicios de rehabilitación, utilización de ayudas ópticas y no ópticas, accesibilidad, oportunidades educativas y laborales. En la población de estudio el uso de lupas y/o bastón de orientación y movilidad fueron las ayudas ópticas y no ópticas, respectivamente, con mayor frecuencia de uso. La falta de suministro de dispositivos ópticos sigue siendo una de las principales causas que contribuye al aumento y sostenimiento de las cifras de discapacidad visual, puesto que genera limitaciones en la actividad y restricciones en la participación ${ }^{2}$. En Colombia los dispositivos de ayudas ópticas y no ópticas entran en una dinámica administrativa compleja entre dispositivos técnicos versus medidas educativas, y algunas entidades promotoras de salud dificultan u obstaculizan su entrega real a los pacientes. Esta realidad contradice parte del marco 
legal en discapacidad en Colombia (para más información, se puede consultar la Ley Estatutaria 1618 de 2013 y el Decreto 1328 de 2016). El informe sobre discapacidad del año $2011^{3}$ es claro en señalar que en el mundo las personas con discapacidad tienen tres veces más probabilidad de que se les niegue la atención en salud (principalmente en el tema de la rehabilitación). Se reporta que las principales razones que contribuyen al aumento de la discapacidad visual son la falta de cobertura social, la falta de participación de las aseguradoras en salud y el poco conocimiento y conciencia en la remisión oportuna de los pacientes con discapacidad visual a un servicio de rehabilitación por parte de los profesionales de la salud ${ }^{47}$.

El presente trabajo tiene limitaciones metodológicas que restringen la extrapolación o validez externa de los resultados; sin embargo, como estudio observacional no analítico, se sugiere la necesidad de realizar estudios con mayor población y de tipo analítico para respaldar que la etiología convencional de la baja visión y la ceguera puede estar cambiando en la actualidad, y sería razonable ajustar los programas de promoción y prevención en discapacidad visual frente a alteraciones de la vía visual y el segmento posterior, con el fin de que se controlen y atiendan con el mismo esmero que los planes de catarata, tamizaje de glaucoma y control de ceguera infecciosa y de ametropías no corregidas.

\section{Conclusiones}

La discapacidad visual es una temática vigente en oftalmología, tanto en el aspecto clínico-práctico como en el académico-investigativo. Según el grado de deficiencia, se subdivide en baja visión y ceguera, y su etiología convencional (principalmente, catarata, glaucoma y ceguera infecciosa) posiblemente esté cambiando como resultado de campañas de promoción y prevención, por fenómenos de transición epidemiológica e incremento de la prevalencia de enfermedades crónicas no transmisibles. Actualmente el espectro del déficit de discapacidad visual parece inclinarse hacia otras entidades que se ubican anatómicamente en el segmento ocular posterior, sin descartar, claro está, la importancia de las enfermedades del segmento anterior y la presencia de procesos neurooftalmológicos (cada vez con mayor frecuencia), por lo que puede ser necesario redireccionar los programas de promoción, prevención y asistencia clínica en baja visión y ceguera.

\section{Agradecimientos}

A cada uno de los integrantes de la línea de investigación en discapacidad del Grupo de Investigación en Discapacidad de la ECS-UPB, así como a los pacientes y familiares que fueron parte de esta investigación.

\section{Responsabilidades éticas}

Protección de personas y animales. Los autores declaran que los procedimientos seguidos se adecuaron a las normas éticas del Comité de Experimentación Humana Responsable y de acuerdo con la Asociación Médica Mundial y la Declaración de Helsinki.

Confidencialidad de los datos. Los autores declaran que siguieron los protocolos de su centro de trabajo sobre la publicación de datos de pacientes.

Derecho a la privacidad y consentimiento informado. Los autores obtuvieron el consentimiento informado de los pacientes y/o sujetos referidos en el artículo. Este documento obra en poder del autor de correspondencia.

\section{Conflicto de intereses}

Los autores no tienen intereses comerciales ni otros conflictos de intereses que declarar.

\section{Financiación}

Centro Integrado para el Desarrollo de la Investigación (CIDI) de la UPB.

\section{Bibliografía}

1. Poma PA. Disability and Culture. Universalism and Diversity. J Natl Med Assoc. 2005;97(3):428

2. OMS. Plan de acción mundial de la OMS sobre discapacidad 2014-2021 [Internet]. Consultado el 29 de enero de 2017. Disponible en: http://www. who.int/disabilities/actionplan/es/.

3. WHO. World report on disability. [Internet]. Consultado el 29 de enero de 2017. Disponible en: http://www.who.int/disabilities/world_report/ 2011/en/.

4. Pan American Health Organization/World Health Organization. OPS OMS. Clasificación de Funcionamiento y Discapacidad. [Internet]. Citado el 29 de enero de 2017. Disponible en: http://www.paho.org/hq/index. php?option=com_content\&view=article\&id=3562\%3A2010-clasificacion-internacional-funcionamiento-discapacidad-salud-cif\&cati$d=511 \% 3$ Ahealth-information-analysis\&ltemid $=2561$ \&lang $=e s$.

5. Stevens GA, White RA, Flaxman SR, Price H, Jonas JB, Keeffe J, et al. Global prevalence of vision impairment and blindness: magnitude and temporal trends, 1990-2010. Ophthalmology. 2013;120(12):2377-84.

6. WHO. Blindness and vision impairment prevention. [Internet]. Consultado el 24 de marzo de 2018. Disponible en: http://www.who.int//blindness/ world sight day/2017/en/\#.

7. Censo general 2005. [Internet]. Consultado el 29 de enero de 2017. Disponible en: https://www.dane.gov.co/index.php/estadisticas-por-tema/ demografia-y-poblacion/censo-general-2005-1.

8. Moreno Angarita M, Ximena Rubio Viscaya S. Realidad y contexto situacional de la población con limitación visual en Colombia: una aproximación desde la justicia y el desarrollo humano. Primera Ed. Bogotá DC: 
Imprenta Braille del Instituto Nacional para Ciegos; 2011. [Internet]. Disponible en: http://www.inci.gov.co.

9. Epidemiología de la Baja Visión en Latinoamérica y Chile. VISIÓN 2020 2013. [Internet]. Consultado el 29 de enero de 2017. Disponible en: https://vision2020la.wordpress.com/2013/11/01/epidemiologia-de-la-baja-vision-en-latinoamerica-y-chile/.

10. WHO. News. [Internet]. Consultado el 29 de enero de 2017. Disponible en: http://www.who.int/blindness/en/.

11. Miqueli-Rodríguez M, López-Hernández SM, Rodríguez-Masó S. Baja visión y envejecimiento de la población. Rev Cuba Oftalmol. 2016;29(3):492-501.

12. van Leeuwen LM, Rainey L, Kef $S$, van Rens GHMB, van Nispen RMA. Investigating rehabilitation needs of visually impaired young adults according to the International Classification of Functioning, Disability and Health. Acta Ophthalmol (Copenh). 2015;93(7):642-50.

13. Ramrattan RS, Wolfs RC, Panda-Jonas S, Jonas JB, Bakker D, Pols HA, et al. Prevalence and causes of visual field loss in the elderly and associations with impairment in daily functioning: the Rotterdam Study. Arch Ophthalmol Chic III 1960. 2001;119(12):1788-94.

14. Stevens GA, White RA, Flaxman SR, Price H, Jonas JB, Keeffe J, et al. Global prevalence of vision impairment and blindness: magnitude and temporal trends, 1990-2010. Ophthalmology. 2013;120(12):2377-84.

15. Oviedo MP, Hernández ML, Ruíz M. Low vision in Colombia: an invisible situation for the country. Rev Fac Nac Salud Pública. 2015;33(1): 22-30.

16. Escudero JC. Discapacidad visual y ceguera en el adulto: revisión de tema. Med UPB. 2011;30(2):170-80.

17. Finger RP, Bertram B, Wolfram C, Holz FG. Blindness and visual impairment in Germany: a slight fall in prevalence. Dtsch Arzteblatt Int 2012;109(27-28):484-9.

18. Resnikoff S, Pascolini D, Etya'ale D, Kocur I, Pararajasegaram R, Pokharel GP, et al. Global data on visual impairment in the year 2002. Bull World Health Organ. 2004;82(11):844-51.

19. Rojas S, Ruiz S, Carvajal J, Álvarez MJ, Duque D, Correa SA, et al Caracterización de una población con discapacidad visual (baja visión y ceguera) atendida en dos Instituciones Prestadoras de Salud de Medellín. Med UPB. 2015;34(1):30-39.

20. Furtado JM, Lansingh VC, Carter MJ, Milanese MF, Peña BN, Ghersi $\mathrm{HA}$, et al. Causes of blindness and visual impairment in Latin America. Surv Ophthalmol. 2012;57(2):149-77.

21. Pathologic Myopia-Clinical Key. [Internet]. Consultado el 29 de enero de 2017. Disponible en: https://www-clinicalkey-es.consultaremota.upb.edu. co/\#!/content/book/3-s2.0-B9781416000167501570.

22. Tano Y. Pathologic myopia: where are we now? Am J Ophthalmol. 2002;134(5):645-60.

23. Pathologic myopia (myopic degeneration) - EyeWiki. [Internet]. Consultado el 29 de enero de 2017. Disponible en: http://eyewiki.aao.org/Pathologic_myopia_(myopic_degeneration).

24. Ohno-Matsui K, Lai TY, Lai CC, Cheung CM. Updates of pathologic myopia. Prog Retin Eye Res. 2016;52:156-87.

25. Yamada M, Hiratsuka Y, Roberts CB, Pezzullo ML, Yates K, Takano S, et al. Prevalence of visual impairment in the adult Japanese population by cause and severity and future projections. Ophthalmic Epidemiol. 2010;17(1):50-7.

26. Cedrone C, Nucci C, Scuderi G, Ricci F, Cerulli A, Culasso F. Prevalence of blindness and low vision in an Italian population: a comparison with other European studies. Eye Lond Engl. 2006;20(6):661-7.

27. Spooner K, Hong T, Wijeyakumar W, Chang AA. Switching to aflibercept among patients with treatment-resistant neovascular age-related macular degeneration: a systematic review with meta-analysis. Clin Ophthalmol. 2017;11:161-77.
28. Pennington $\mathrm{KL}$, DeAngelis $\mathrm{MM}$. Epidemiology of age-related macular degeneration (AMD): associations with cardiovascular disease phenotypes and lipid factors. Eye Vis (Lond). 2016;3:34.

29. Wong WL, Su X, Li X, Cheung CM, Klein R, Cheng CY, et al. Global prevalence of age-related macular degeneration and disease burden projection for 2020 and 2040: a systematic review and meta-analysis. Lancet Glob Health. 2014;2(2):e106-116.

30. Eduardo Leite Arieta C, Nicolini Delgado AM, José NK, Temporini ER, Alves MR, de Carvalho Moreira Filho D. Refractive errors and cataract as causes of visual impairment in Brazil. Ophthalmic Epidemiol. 2003:10(1):15-22.

31. Cass H, Landers J, Benitez P. Causes of blindness among hospital outpatients in Ecuador. Clin Experiment Ophthalmol. 2006;34(2):146-51.

32. Strong S, Liew G, Michaelides M. Retinitis pigmentosa-associated cystoid macular oedema: pathogenesis and avenues of intervention. $\mathrm{Br} \mathrm{J} O p h-$ thalmol. 2017;101(1):31-7.

33. Zhang Q. Retinitis Pigmentosa: Progress and Perspective. Asia-Pac J Ophthalmol Phila Pa. 2016;5(4):265-71.

34. Hartong DT, Berson EL, Dryja TP. Retinitis pigmentosa. Lancet Lond Engl. 2006;368(9549):1795-809.

35. Prevalencia de ceguera en Colombia. Estudio poblacional en una comunidad urbana. 26/2/2004. [Internet]. Consultado el 29 de enero de 2017. Disponible en: http://www.pagina-digital.com.ar/articulos/2004/2004prim/ tecnologia2/vis24-2pl.asp.

36. Prevalencia de ceguera en el Departamento de Santander - Colombia ResearchGate. [Internet]. Consultado el 29 de enero de 2017. Disponible en: https://www.researchgate.net/publication/26848459_Prevalencia_de_ ceguera en el Departamento de Santander - Colombia. «

37. Rodríguez FJ, Brieke AC, Guerrero J, Porras A, Zarabanda MP. Prevalencia de retinopatía diabética en consulta privada en Bogotá, Colombia desde el 2000 hasta el 2007. Rev Soc Colomb Oftalmol. 2009;42(2):197204.

38. Zuluaga C, Sierra MV, Asprilla E. Causas de ceguera infantil en Cali, Colombia. Colombia Médica. 2005. [Internet]. Consultado el 29 de enero de 2017. Disponible en: http://www.redalyc.org/articulo.oa?id=28336402.

39. Cañón Cárdenas YZ. La baja visión en Colombia y en el mundo. Cien Tecnol Salud Vis Ocul. 2011;9(1):117-23.

40. Suárez-Escudero JC. Discapacidad y neurociencias: la mağnitud del déficit neurológico y neuropsiquiátrico. Acta Neurológica Colomb. 2014;30(4):290-9.

41. Quillen DA. Common causes of vision loss in elderly patients. Am Fam Physician. 1999;60(1):99-108.

42. Pascolini D, Mariotti SP. Global estimates of visual impairment: $2010 . \mathrm{Br}$ J Ophthalmol. 2012;96(5):614-8.

43. Abou-Gareeb I, Lewallen S, Bassett K, Courtright P. Gender and blindness: a meta-analysis of population-based prevalence surveys. Ophthalmic Epidemiol. 2001;8(1):39-56.

44. Olatunji VA, Adepoju FG, Owoeye JF. Perception and Attitude of a Rural Community Regarding Adult Blindness in North Central Nigeria. Middle East Afr J Ophthalmol. 2015;22(4):508-13.

45. Yonekawa Y, Varma R, Choudhury F, Torres M, Azen SP, Los Angeles Latino Eye Study Group. Risk factors for four-year incident visual impairment and blindness: the Los Angeles Latino Eye Study. Ophthalmology. 2011;118(9):1790-7.

46. Liu JH, Cheng CY, Chen SJ, Lee FL. Visual impairment in a Taiwanese population: prevalence, causes, and socioeconomic factors. Ophthalmic Epidemiol. 2001;8(5):339-50

47. Centers for Disease Control and Prevention (CDC). Reasons for not seeking eye care among adults aged $\geq 40$ years with moderate-to-severe visual impairment--21 States, 2006-2009. MMWR Morb Mortal Wkly Rep. 2011;60(19):610-3. 